\title{
Adaptive Backstepping Compensation of Drives with Sandwiched Deadzone Nonlinearity
}

\author{
Nizar J. Alkhateeb*, Hameed K. Ebraheem, Ebraheem K. Sultan, Bassam M. Alrahsed \\ Department of Electronic Engineering Technology, College of Technological Studies, Public Authority of Applied Education and \\ Training (PAAET), Shuwaikh, Kuwait \\ Email: ${ }^{\star}$ nj.alkhateeb@paaet.edu.kw
}

How to cite this paper: Alkhateeb, N.J., Ebraheem, H.K., Sultan, E.K. and Alrahsed, B.M. (2021) Adaptive Backstepping Compensation of Drives with Sandwiched Deadzone Nonlinearity. Intelligent Control and Automation, 12, 73-89.

https://doi.org/10.4236/ica.2021.123005

Received: May 1, 2021

Accepted: July 26, 2021

Published: July 29, 2021

Copyright (C) 2021 by author(s) and Scientific Research Publishing Inc. This work is licensed under the Creative Commons Attribution International License (CC BY 4.0).

http://creativecommons.org/licenses/by/4.0/

\begin{abstract}
In this paper, a nonlinear robust adaptive controller is proposed for gear transmission servo system (GTS) containing a sandwiched deadzone due to improper gear meshing. The controller is robust to dynamic uncertainties and can compensate the effect caused by the sandwiched nonlinearity which is separated from the control input through drive compliance. The proposed design methodology does not require an adaptive inverse deadzone function and does not require the knowledge of its parameter and only the knowledge of upper bounds is required.
\end{abstract}

\section{Keywords}

Sandwiched Deadzone, Adaptive Control, Nonlinearity, Lyapunov Stability

\section{Introduction}

Deadzone is one of the most common nonlinearities that affect a typical gear transmission servo systems (GTS) where the spacing between meshing causes a temporary loss of contact resulting in an inevitable mismatch error between desired reference trajectory and the actual one. It has been shown that if the problem is not addressed properly, the output of the system exhibits limit cycles which affect the accuracy of the overall system dynamics especially in applications requiring high precision [1]. In general, deadzone may result in oscillations or even overall system instability if it is not accounted for. The task of compensating for such errors is complicated further by the fact that the classical modeling for the deadzone nonlinearity is discontinuous making it non-differentiable. Sandwich deadzone nonlinearity exacerbates the problem even more because the nonlinearity falls in the middle of two different dynamic subsystems. Some of the common systems with sandwiched deadzone are hydraulic actuators, servome- 
chanism position control systems with gears, and piezoelectric transducers which have a hysteresis in their characteristic. Inverse model compensation improved performance by greatly reducing tracking errors [2] [3] [4]. Nonetheless, inverse compensation scheme requires instantaneous jumps to traverse the inner deadzone segment which is not possible for systems with inertia such as mechanical gears. Several researchers have applied variable structure or sliding mode controllers for systems with input deadzone [5] [6]. Although the overall system was shown to be stable the problem of chattering persisted and further complicated the problem. In [7], a robust sliding mode controller has been designed to ensure the convergence of the system trajectories in a neighborhood of the origin of arbitrary width (independently of the deadzone size). In a more recent work [1], a new output tracking backstepping algorithm for GTS subject to backlash nonlinearity was developed. To overcome the nondifferentiability of deadzone the authors developed a soft differentiable model of deadzone which allowed them to analytically prove the stability of the overall system [8] and simulations showed the elimination limit cycling problem and improved tracking performance. In [9] a cascade system was presented and controlled by using smooth dynamic feedback with two constraint conditions were used to prove the global stability of a soft sandwich deadzone. To address the tracking problem for sandwich-like systems, Taware et al. [10] first proposed an inner-outer loop structured controller with a nonlinearity inverse. Furthermore, in a different study [11], Taware and Tao applied the adaptive technique to the unknown deadzone case. An adaptive hybrid control scheme for a sandwiched deadzone systems was presented in [12]. The proposed control scheme employs an inner-loop discrete-time feedback design and an outer-loop continuous-time feedback design, combined with an adaptive dead-zone inverse to reduce the dead-zone effect resulting in improved output tracking. The study proposed a bounded tracking control of a sandwiched system with a nonsymmetric deadzone nonlinearity coupled with a saturation constraint to achieve acceptable output tracking results. In [13] a semiglobal stabilization of sandwich systems by dynamic output feedback. The authors state that "the controller is a mathematical construction that is not intended for practical implementation in its current form". Wang, et al. in [14] considered a class of uncertain dynamic nonlinear systems preceded by unknown dead-zone nonlinearities, in the presence of bounded external disturbances. Employing robust adaptive backstepping control algorithms without requiring the uncertain parameters within known intervals. The proposed controllers guarantee global stability as well as certain transient performance. A similar problem was proposed by [10] to control a sandwich non-smooth nonlinearities between linear dynamic blocks by employing an inner-loop discrete-time feedback design along with an outer-loop continuous-time feedback design, combined with a nonlinearity inverse, to cancel the nonlinearity effect, for improving output tracking. In a more recent study, Azhdari and Binzadeh addressed the problem of combined sandwiched deadzone and saturation non- 
linerities with unknown parameters and time varying external disturbances [15]. They reported success in achieving boundedness of the overall closed loop system with simulations provided as proof of concept. The idea to treat the deleterious effect of deadzone motivated us to approach the tenuous task of controling a hard nonlinearity in a sandwiched setting. The main contribution of this paper is the application of an adaptive robust controller without the need to soften the hard deadzone model. In our previously published paper [2], a robust adaptive nonlinear controller to handle the problem of input deadzone without the need of a deadzone inverse block was successfully developed and applied. In this paper, we extend the application of the robust adaptive controller to address the sandwich deadzone problem by utilizing adaptive backstepping. In Section 4, a review of the overall system dynamics with a sandwiched deadzone nonlinearity and presenting a modified controller. The modified controller will be used in applying the backstepping technique to reach the final form of the robust controller. Simulation studies show a clear improvement in the tracking and step response of the overall system.

\section{Problem Statement: Incorporating Drive-Train Compliance in the Design}

In many applications, compliance is present in the drive system. This leads to another level of complication since the deadzone nonlinearity is present both on the motor and the load side and is not matched with the control input. The control design developed in [2] can be extended to the case when there is a compliance in the drive-train with the deadzone acting on both the actuator and load ends. The dynamics of a GTS system with sandwiched deadzone may be written as

$$
\begin{gathered}
J_{m} \frac{\mathrm{d}^{2} \theta_{m}}{\mathrm{~d} t^{2}}+c_{m} \frac{\mathrm{d} \theta_{m}}{\mathrm{~d} t}=\tau-\mathrm{DZ}\left(\theta_{r}, \delta, m\right) \\
J_{l} \frac{\mathrm{d}^{2} \theta_{l}}{\mathrm{~d} t^{2}}+c_{l} \frac{\mathrm{d} \theta_{l}}{\mathrm{~d} t}=N_{0} \cdot \operatorname{DZ}\left(\theta_{r}, \delta, m\right),
\end{gathered}
$$

where $J_{l}, c_{l}$ and $\theta_{l}$ are the moment of inertia, viscous friction coefficient, and angle position on the load side respectively; similarly, $J_{m}, c_{m}$ and $\theta_{m}$ are the perspective variables on the driving side. In addition, $m$ is the rigidity constant, and $2 \delta$ is the width of the deadzone spacing. Moreover, $N_{0}$ defines the gear ratio, and $\tau$ is the driving torque. Meanwhile, $\theta_{r}$ is the relative displacement parameter is given by

$$
\theta_{r}=\theta_{m}-N_{0} \theta_{l},
$$

is utilized in the deadzone function $\mathrm{DZ}(\cdot)$, commonly written as

$$
\mathrm{DZ}\left(\theta_{r}, \delta, m\right)= \begin{cases}m\left(\theta_{r}-\delta\right), & \text { if } \theta_{r}>\delta \\ 0, & \text { if }-\delta<\theta_{r}<\delta \\ m\left(\theta_{r}+\delta\right), & \text { if } \theta_{r}<-\delta\end{cases}
$$


In general, another useful representation of the deadzone function is written as

$$
\operatorname{DZ}(\theta)=m\left[\theta_{r}-\operatorname{Sat}\left(\theta_{r}\right)\right]
$$

where $\operatorname{Sat}(\cdot)$ represents the saturation function defined as

$$
\operatorname{Sat}\left(\theta_{r}\right)= \begin{cases}\delta, & \text { if } \theta_{r}>\delta \\ \theta_{r}, & \text { if }-\delta<\theta_{r}<\delta \\ -\delta, & \text { if } \theta_{r}<-\delta\end{cases}
$$

The function $\psi\left(\theta_{r}\right)$ is defined to represent the normalized saturation function as follows

$$
\psi\left(\theta_{r}\right)= \begin{cases}1, & \text { if } \theta_{r}>\delta \\ \theta_{r} / \delta, & \text { if }-\delta<\theta_{r}<\delta \\ -1, & \text { if } \theta_{r}<-\delta\end{cases}
$$

Hence, the deadzone nonlinearity function may be written more compactly as

$$
\mathrm{DZ}(\theta)=m\left(\theta_{r}-\delta \psi\left(\theta_{r}\right)\right)
$$

An important and useful quality of the normalized saturation function $\left\|\psi\left(\theta_{r}\right)\right\|$ that will be employed in the ensuing compensator design is stated in the following property:

Property 1 . If at $t=t_{0}$, the normalized saturation function $\psi\left(\theta_{r}\left(t_{0}\right)\right) \in[-1,1]$ then $\left|\psi\left(\theta_{r}\right)\right| \leq 1 \Rightarrow \forall t \geq 0$.

Basically, the property sets an upper bounds equal to $\delta$ on the saturation function $\operatorname{Sat}\left(\theta_{r}\right)$ because $\left|\psi\left(\theta_{r}\right)\right| \leq 1$. Meanwhile, writing the state space representation for the GTS system described in (1) and (2) combined with equations (3) and (8) yields

$$
\begin{gathered}
\dot{\theta}_{l}=\omega_{l} \\
\dot{\omega}_{l}=\frac{1}{J_{l}}\left\{-c_{l} \omega_{l}+m\left(\theta_{m}-N_{0} \theta_{l}\right)-m \delta \psi\left(\theta_{r}\right)\right\} \\
\dot{\theta}_{m}=\omega_{m} \\
\dot{\omega}_{m}=\frac{1}{J_{m}}\left\{-c_{m} \omega_{m}+\tau-m\left(\theta_{m}-N_{0} \theta_{l}\right)+m \delta \psi\left(\theta_{r}\right)\right\} .
\end{gathered}
$$

It is noteworthy that the system dynamics given by (9) are not in any of the standard canonical forms for which the backstepping methodology is readily applicable. However, through the application of the saturation function property combined with the strengthened results of Lemma RANDM [16] and [17], the compensator design is carried out along similar lines as the systematic design procedure presented.

\section{Applying Adaptive Backstepping Control Method}

Based on the results presented in [2], and by looking at the load gear subsystem dynamics

$$
\dot{\theta}_{l}=\omega_{l}
$$




$$
\dot{\omega}_{l}=-\frac{c_{l}}{J_{l}} \omega_{l}+\frac{m}{J_{l}} \cdot\left\{\left(\theta_{m}-N_{0} \theta_{l}\right)-\delta \psi\left(\theta_{r}\right)\right\}
$$

can be rewritten as

$$
\dot{x}=\left[\begin{array}{c}
\dot{\theta}_{l} \\
\dot{\omega}_{l}
\end{array}\right]=A x+B\left\{\theta_{m}-\delta \psi\left(\theta_{r}\right)\right\}
$$

where

$$
x=\left[\begin{array}{c}
\theta_{l} \\
\omega_{l}
\end{array}\right], \quad A=\left[\begin{array}{cc}
0 & 1 \\
\frac{-m N_{0}}{J_{l}} & \frac{-c_{l}}{J_{l}}
\end{array}\right], \quad B=\left[\begin{array}{c}
0 \\
\frac{m}{J_{l}}
\end{array}\right],
$$

moreover, the normalized saturation function $\psi\left(\theta_{r}\right)$ is treated as a bounded unmeasurable disturbance by virtue of Property (1). The procedure begins with designing the control law by assuming that $\theta_{m}$ is the virtual control for the load subsystem 33. Since $\theta_{m}$ is not the actual control, we define the error between $\theta_{m}$ and the virtual control developed, and design $\omega_{m}$ appearing in the dynamics for this error such that the error goes to zero asymptotically. Thus, virtual controls are designed for $\theta_{m}$ and $\omega_{m}$, and finally the actual control input $\tau$ is designed for the overall system. The details of the procedure are presented in the following steps:

Step 0: Note that the load side subsystem dynamics are not in the output canonical form (since the saturation function $\psi\left(\theta_{r}\right)$ is not measurable) for which the technique of backstepping can be applied directly. Initially, the motor angle $\theta_{m}$ is treated as the virtual control for the $x=\left[\begin{array}{ll}\theta_{l} & \omega_{l}\end{array}\right]$ subsystem. In this step, the result presented in [2] which is basically a strengthend version of the previously proven Lemma RANDM in [16] [17] is employed. The lemma is modified though for the special regulation case for the sake of clarity. Furthermore, based on the result of [2], the virtual control law for the subsystem 33 is written as

$$
\theta_{m}^{*}=-\alpha B^{\mathrm{T}} P x-\hat{\beta} B^{\mathrm{T}} P x-\rho_{l} \operatorname{Tanh}\left[(a+b t) B^{\mathrm{T}} P x\right]
$$

where $\alpha, a, b>0, \rho_{l} \geq 2 \delta$; while $P$ is a positive definite, symmetric solution to the Algebraic Riccati Equation (ARE)

$$
A^{\mathrm{T}} P+P A-2 \hat{\alpha} P B B^{\mathrm{T}} P+Q=0
$$

with $Q$ a symmetric positive definite matrix, and $\hat{\beta}$ is the adaptation parameter with dynamics given by

$$
\dot{\hat{\beta}}=\Gamma\left\|B^{\mathrm{T}} P x\right\|^{2}
$$

with the constant $\Gamma>0$. The properties of the virtual control law are stated in the following theorem:

Theorem 4.1: The closed-loop subsystem (11) under the smooth, robust virtual controller (12) and the adaptation (14), is globally asymptotically stable. Furthermore, the position error asymptotically converges to zero. 
Proof: The stability properties of the above virtual control law using the Lyapunov function

$$
V_{0}=x^{\mathrm{T}} P x+\frac{1}{2} \Gamma^{-1}\left(\hat{\beta}-\beta^{*}\right)^{2} .
$$

Differentiating $V_{0}$ along the trajectories of the closed-loop system, we obtain

$$
\begin{aligned}
\dot{V}_{0}= & \dot{x}^{\mathrm{T}} P x+x^{\mathrm{T}} P \dot{x}+\left(\hat{\beta}-\beta^{*}\right) \dot{\hat{\beta}} \\
= & {\left[A x+B\left(\theta_{m}-\delta \psi\left(\theta_{r}\right)\right)\right]^{\mathrm{T}} P x } \\
& +x^{\mathrm{T}} P\left[A x+B\left(\theta_{m}-\delta \psi\left(\theta_{r}\right)\right)\right]+\left(\hat{\beta}-\beta^{*}\right)\left\|B^{\mathrm{T}} P x\right\|^{2} .
\end{aligned}
$$

Replacing $\theta_{m}=\tilde{\theta}_{m}-\theta_{m}^{*}$ and applying the virtual control law $\theta_{m}^{*}$ given in (12) and the adaptation law (14) yields

$$
\begin{aligned}
\dot{V}_{0}= & {\left[A x+B\left(-\alpha B^{\mathrm{T}} P x-\hat{\beta} B^{\mathrm{T}} P x-\rho_{l} \operatorname{Tanh}\left[(a+b t) B^{\mathrm{T}} P x\right]-\delta \psi\left(\theta_{r}\right)\right)\right]^{\mathrm{T}} P x } \\
& +2 x^{\mathrm{T}} P B \tilde{\theta}_{m}+x^{\mathrm{T}} P\left[A x+B\left(-\alpha B^{\mathrm{T}} P x-\hat{\beta} B^{\mathrm{T}} P x\right.\right. \\
& \left.\left.-\rho_{l} \operatorname{Tanh}\left[(a+b t) B^{\mathrm{T}} P x\right]-\delta \psi\left(\theta_{r}\right)\right)\right]+\left(\hat{\beta}-\beta^{*}\right)\left\|B^{\mathrm{T}} P x\right\|^{2} \\
= & x^{\mathrm{T}} A^{\mathrm{T}} P X-\alpha x^{\mathrm{T}} P B B^{\mathrm{T}} P X+x^{\mathrm{T}} P A x+\left(\hat{\beta}-\beta^{*}\right)\left\|B^{\mathrm{T}} P x\right\|^{2}-\hat{\beta} x^{\mathrm{T}} P B B^{\mathrm{T}} P x \\
& +2\left(x^{\mathrm{T}} P B\right) \tilde{\theta}_{m}-2 \rho_{l}\left(x^{\mathrm{T}} P B\right) \operatorname{Tanh}\left[(a+b t) B^{\mathrm{T}} P x\right]-2 \delta x^{\mathrm{T}} P B \psi\left(\theta_{r}\right) .
\end{aligned}
$$

In the last equation, the term $\hat{\beta} x^{\mathrm{T}} P B B^{\mathrm{T}} P x$ is equal to the term $\hat{\beta}\left\|B^{\mathrm{T}} P x\right\|^{2}$ and therefore the two terms are eliminated. Once again, by rearranging the terms in the last equation results in

$$
\begin{aligned}
\dot{V}_{0}= & x^{\mathrm{T}}\left(A^{\mathrm{T}} P+P A-\alpha P B B^{\mathrm{T}} P\right) x-\beta^{*}\left\|B^{\mathrm{T}} P x\right\|^{2}+2 x^{\mathrm{T}} P B \tilde{\theta}_{m} \\
& -2 x^{\mathrm{T}} P B\left\{\rho_{l} \operatorname{Tanh}\left[(a+b t) B^{\mathrm{T}} P x\right]-\delta \psi\left(\theta_{r}\right)\right\} .
\end{aligned}
$$

Collecting terms and utilizing the solution of the ARE (13) results in

$$
\begin{aligned}
\dot{V}_{0}= & -x^{\mathrm{T}} Q x-\beta^{*}\left\|B^{\mathrm{T}} P x\right\|^{2}+2 x^{\mathrm{T}} P B \tilde{\theta}_{m} \\
& -2 x^{\mathrm{T}} P B\left[\rho_{l} \operatorname{Tanh}\left[(a+b t) B^{\mathrm{T}} P x\right]+\delta \psi\left(\theta_{r}\right)\right] .
\end{aligned}
$$

Applying this bound obtains

$$
\begin{aligned}
\dot{V}_{0} \leq & -\lambda_{\text {min }}(Q)\|x\|^{2}-\beta^{*}\left\|x^{\mathrm{T}} P B\right\|^{2}+2 x^{\mathrm{T}} P B \tilde{\theta}_{m} \\
& -2 x^{\mathrm{T}} P B\left\{\rho_{l} \operatorname{Tanh}\left[(a+b t) B^{\mathrm{T}} P x\right]+\delta \psi\left(\theta_{r}\right)\right\} .
\end{aligned}
$$

The first two terms are negative while the third term will be taken care in the next step of the design process. As for the last term in (20), to ensure that $\dot{V}_{0} \leq 0$ we have to examine the condition needed to properly negate its overall outcome. To begin with the term can be bounded as

$$
\begin{aligned}
& -2 x^{\mathrm{T}} P B\left\{\rho_{l} \operatorname{Tanh}\left[(a+b t) B^{T} P x\right]+\delta \psi\left(\theta_{r}\right)\right\} \\
& =-\rho_{l} x^{\mathrm{T}} P B\left\{\frac{\delta}{\rho_{l}} \psi\left(\theta_{r}\right)+\operatorname{Tanh}\left[(a+b t) B^{\mathrm{T}} P x\right]\right\} .
\end{aligned}
$$


In order for the term to be negative the following inequality, along with the fact that the upper bounds on the normalized saturation function (7) as $\left\|\psi\left(\theta_{r}\right)\right\| \leq 1$, and the Tan hyperbolic function has an upper bound of 1 , yields the condition

$$
\begin{gathered}
\frac{\delta}{\rho_{l}}\left\|\psi\left(\theta_{r}\right)\right\|<\left\|\operatorname{Tanh}\left[(a+b t) B^{\mathrm{T}} P x\right]\right\| \\
\frac{\delta}{\rho_{l}}<1 .
\end{gathered}
$$

Hence, by properly selecting the gain parameter $\rho_{l}$ to be greater than $\delta$ ensures $\dot{V}_{0} \leq 0$ making the subsystem asymptotically stable; thereby, concluding the proof of Theorem 4.1.

Step 1: Since $\theta_{m}$ is not the actual control, an error $\tilde{\theta}_{m}=\theta_{m}-\theta_{m}^{*}$ is established and differentiated to obtain the dynamics for $\tilde{\theta}_{m}$ which can be written as:

$$
\begin{aligned}
\dot{\tilde{\theta}}_{m}=\omega_{m}-\frac{\partial \theta_{m}^{*}}{\partial x}\left[A x+B\left\{\theta_{m}-m \delta \psi\left(\theta_{r}\right)\right\}\right]-\frac{\partial \theta_{m}^{*}}{\partial t}-\frac{\partial \theta_{m}^{*}}{\partial \hat{\beta}} \dot{\hat{\beta}} \\
\triangleq \omega_{m}-\chi_{1}(x, \hat{\beta})-\gamma_{1}(x, \hat{\beta}) m \delta \psi\left(\theta_{r}\right) .
\end{aligned}
$$

To derive the stabilizing virtual controller for the extended subsystem that inculdes $\left[\begin{array}{cc}x & \tilde{\theta}_{m}\end{array}\right]$ a newly formed Lyapunov function is proposed. A virtual contoller will be porposed to ensure the over all stability properties of the subsystem. Consider

$$
V_{1}=V_{0}+\frac{1}{2} \tilde{\theta}_{m}^{2}=x^{\mathrm{T}} P x+\frac{1}{2} \Gamma^{-1}\left(\hat{\beta}-\beta^{*}\right)^{2}+\frac{1}{2} \tilde{\theta}_{m}^{2} .
$$

Differentiating $V_{1}$ along the trajectories of the $\left[\begin{array}{cc}x & \tilde{\theta}_{m}\end{array}\right]$ subsystem, one obtains

$$
\begin{aligned}
\dot{V}_{1}= & \dot{V}_{0}+\tilde{\theta}_{m} \cdot \dot{\tilde{\theta}}_{m}=\dot{V}_{0}+\tilde{\theta}_{m}\left(\dot{\theta}_{m}-\dot{\theta}_{m}^{*}\right)+2 x^{\mathrm{T}} P B \tilde{\theta}_{m} \\
& =\dot{V}_{0}+\tilde{\theta}_{m} \cdot\left\{\omega_{m}-\omega_{m}^{*}+2 x^{\mathrm{T}} P B\right\},
\end{aligned}
$$

where

$$
\omega_{m}^{*}=\dot{\theta}_{m}^{*}=\frac{\partial \theta_{m}^{*}}{\partial x} \dot{x}+\frac{\partial \theta_{m}^{*}}{\partial \hat{\beta}} \dot{\hat{\beta}}+\frac{\partial \theta_{m}^{*}}{\partial t} \triangleq \chi_{1}(x, \hat{\beta})+\gamma_{1}(x, \hat{\beta}) \operatorname{Sat}\left(\theta_{m}-N_{0} \theta_{l}\right) .
$$

Refer to the appendix for complete derivations of the functions $\chi_{1}(x, \hat{\beta})$ and $\gamma_{1}(x, \hat{\beta})$. Subsequently, $\omega_{m}$ is now viewed as the virtual control for the $\left[\begin{array}{cc}x & \tilde{\theta}_{m}\end{array}\right]$ subsystem. The virtual control for $\omega_{m}^{*}$ is given by

$$
\omega_{m}^{*}=-\sigma_{0} \tilde{\theta}_{m}-2 x^{\mathrm{T}} P B+\chi_{1}(x, \hat{\beta})-\rho_{l} \gamma_{1}(x, \hat{\beta}) \operatorname{Tanh}\left[(a+b t) \gamma_{1}(x, \hat{\beta}) \tilde{\theta}_{m}\right]
$$

where $\sigma_{0}>0$.

$\dot{V}_{1} \leq \dot{V}_{0}-\sigma_{0} \tilde{\theta}_{m}^{2}-\tilde{\theta}_{m}\left[\rho_{l} \gamma_{1}(x, \hat{\beta}) \operatorname{Tanh}\left[(a+b t) \gamma_{1}(x, \hat{\beta}) \tilde{\theta}_{m}\right]+\gamma_{1}(x, \hat{\beta}) \delta \psi\left(\theta_{r}\right)\right]$.

the last term can be handled in a similar manner as in the conclusion of the 
proof of Theorem 4.1. By rewriting the last term as

$$
\begin{gathered}
-\tilde{\theta}_{m} \rho_{l} \gamma_{1}(x, \hat{\beta})\left[\operatorname{Tanh}\left[(a+b t) \gamma_{1}(x, \hat{\beta}) \tilde{\theta}_{m}\right]+\frac{\delta}{\rho_{l}} \psi\left(\theta_{r}\right)\right], \\
-\left\|\tilde{\theta}_{m} \rho_{l} \gamma_{1}(x, \hat{\beta})\right\|\left[\operatorname{Tanh}\left[(a+b t)\left\|\gamma_{1}(x, \hat{\beta}) \tilde{\theta}_{m}\right\|\right]+\frac{\delta}{\rho_{l}}\left\|\psi\left(\theta_{r}\right)\right\|\right],
\end{gathered}
$$

shows that by ensuring that the gain parameter $\rho_{l}$ to be greater than $\delta$ ensures $\dot{V}_{1} \leq 0$. Employing the same arguments as in the proof of Theorem 4.1, it follows that $\left\|x^{\mathrm{T}} P B\right\|$ and $\tilde{\theta}_{m}$ converge asymptotically to zero. However, since $\omega_{m}$ given in Equation (26) is not the actual control, the error is defined as

$$
\tilde{\omega}_{m}=\omega_{m}-\omega_{m}^{*}
$$

and the dynamics of $\tilde{\omega}_{m}$ can be obtained through differentiation as

$$
\begin{gathered}
\dot{\omega}_{m}^{*}=\frac{\partial \omega_{m}^{*}}{\partial x} \dot{x}+\frac{\partial \omega_{m}^{*}}{\partial \hat{\beta}} \dot{\hat{\beta}}+\frac{\partial \omega_{m}^{*}}{\partial t} \triangleq \chi_{2}\left(x, \tilde{\theta}_{m}, \hat{\beta}\right)+\gamma_{2}\left(x, \tilde{\theta}_{m}, \hat{\beta}\right) \operatorname{Sat}\left(\theta_{m}-\theta_{l}\right) \\
\dot{\tilde{\omega}}_{m}=\frac{1}{J_{m}}\left\{\tau-c_{m} \omega_{m}-\left(\theta_{m}-N_{0} \theta_{l}\right)+\delta \psi\left(\theta_{r}\right)\right\} \\
-\chi_{2}\left(x, \tilde{\theta}_{m}, \hat{\beta}\right)-\gamma_{2}\left(x, \tilde{\theta}_{m}, \hat{\beta}\right) \delta \psi\left(\theta_{r}\right)
\end{gathered}
$$

where $\chi_{2}$ and $\gamma_{2}$ are completely derived and presented in the appendix. The actual control $\tau$ now appears in $\tilde{\omega}_{m}$ dynamics which is designed in the next step.

Step 2: The Lyapunov function used for analysis of the overall closed-loop system is given by

$$
V_{2}=V_{1}+\frac{1}{2} \tilde{\omega}_{m}^{2}=x^{\mathrm{T}} P x+\frac{1}{2} \Gamma^{-1}\left(\hat{\beta}-\beta^{*}\right)^{2}+\frac{1}{2} \tilde{\theta}_{m}^{2}+\frac{1}{2} \tilde{\omega}_{m}^{2} .
$$

The following robust control law is developed for the overall system:

$$
\begin{aligned}
\tau= & -\sigma_{2} \tilde{\omega}_{m}-\left(\theta_{m}-N_{0} \theta_{l}\right)-\rho_{m} \operatorname{Tanh}\left[(a+b t) \tilde{\omega}_{m}\right]+\chi_{2}\left(x, \tilde{\theta}_{m}, \hat{\beta}\right) \\
& +\rho_{l} \gamma_{2}\left(x, \tilde{\theta}_{m}, \hat{\beta}\right) \operatorname{Tanh}\left[(a+b t) \gamma_{2}\left(x, \tilde{\theta}_{m}, \hat{\beta}\right) \tilde{\omega}_{m}\right],
\end{aligned}
$$

where $\sigma_{2}>0$ and the $\rho_{m}>2 \delta$ is incorporated to compensate for the deadzone effect on the actuator side. Differentiating $V_{2}$ along the trajectories of the overall system yields

$$
\begin{aligned}
\dot{V}_{2} \leq & -\lambda_{\min }(Q)\|x\|^{2}-\beta^{*}\left(x^{\mathrm{T}} P B\right)^{2}-\sigma \tilde{\theta}_{m}^{2}-\sigma_{2} \tilde{\omega}_{m}^{2} \\
& -\tilde{\omega}_{m}\left\{\rho_{m} \operatorname{Tanh}\left[(a+b t) \tilde{\omega}_{m}\right]-\delta \psi\left(\theta_{r}\right)\right\} \\
& -\tilde{\omega}_{m}\left\{\rho_{l} \gamma_{2}\left(x, \tilde{\theta}_{m}, \hat{\beta}\right) \operatorname{Tanh}\left[(a+b t) \gamma_{2}\left(x, \tilde{\theta}_{m}, \hat{\beta}\right) \tilde{\omega}_{m}\right]\right. \\
& \left.-\gamma_{2}\left(x, \tilde{\theta}_{m}, \hat{\beta}\right) \mathrm{d} \psi\left(\theta_{r}\right)\right\} .
\end{aligned}
$$

Citing the same arguments used in the proof of Theorem 4.1, both gain parameters $\rho_{l}$ and $\rho_{m}$ must satisfy the following two conditions:

$$
\rho_{l}>2 \delta \rho_{m}>2 \delta
$$


to ensure all terms in $V_{2}<0$. Accordingly, the terms $\left\|x^{\mathrm{T}} P B\right\|, \tilde{\theta}_{m}$, and $\tilde{\omega}_{m}$ converge asymptotically to zero. In conclusion, the result of the proof is summarized in the following theorem.

Theorem 4.2. Asymptotic convergence to zero for the load position errors for the system (9) under the robust smooth controller (30) with the adaptation update law (14) is achieved and only upper bounds on the deadzone spacing is required.

\section{Simulation Studies}

Simulations were performed to demonstrate the efficacy of the designed controller (30). The parameters for a typical GTS system, described by the dynamic system Equations (9), are presented in Table 1.

In addition, Listed in Table 2 are the constant parameters for the designed controller. These parameters provide a good basis for simulation studies to elucidate the efficacy of our control design methodology. Although, the parameters reflect a standard gear transmission system it is not linked to any real experimental test bed.

Based on the parameters listed in Table 1 and Table 2, the load gear subsystem dynamics

$$
\begin{gathered}
\dot{\theta}_{l}=\omega_{l} \\
\dot{\omega}_{l}=-0.24 \omega_{l}+0.40\left\{\left(\theta_{m}-5.0 \theta_{l}\right)-\delta \psi\left(\theta_{r}\right)\right\}
\end{gathered}
$$

Table 1. System parameters.

\begin{tabular}{ccc}
\hline & Parameters of the GTS & \\
\hline Inertia of the Motor & $J_{m}$ & $0.01 \mathrm{~kg} \cdot \mathrm{m}^{2}$ \\
Inertia of the Load & $J_{l}$ & $0.5 \mathrm{~kg} \cdot \mathrm{m}^{2}$ \\
Deadzone Spacing & $\delta$ & $0.02 \mathrm{Rad}$ \\
Gear Ratio & $N_{0}$ & $5 ; \mathrm{none}$ \\
Rigidity constant & $m$ & $0.2 ; \mathrm{Nm} / \mathrm{rad}$ \\
Load Viscous Friction & $c_{l}$ & $0.12 ; \mathrm{Nm} / \mathrm{rad}$ \\
Motor Viscous Friction & $c_{m}$ & $0.12 ; \mathrm{Nm} / \mathrm{rad}$ \\
\hline
\end{tabular}

Table 2. Controller design parameters used in the simulations.

\begin{tabular}{cc}
\hline Parameters & Value \\
\hline$\alpha$ & 1.0 \\
$\sigma_{0}$ & 1.0 \\
$\sigma_{2}$ & 1.0 \\
$\rho_{l}$ & 2.0 \\
$\rho_{m}$ & 2.0 \\
$(a, b)$ & $(10,100)$ \\
$\Gamma$ & 500
\end{tabular}




$$
\begin{gathered}
\dot{\theta}_{m}=\omega_{m} \\
\dot{\omega}_{m}=-12.0 \omega_{m}+\tau-0.40\left\{\left(\theta_{m}-5.0 \theta_{l}\right)+\delta \psi\left(\theta_{r}\right)\right\},
\end{gathered}
$$

which can compactly be rewritten as

$$
\dot{x}=\left[\begin{array}{c}
\dot{\theta}_{l} \\
\dot{\omega}_{l}
\end{array}\right]=A x+B\left\{\theta_{m}-0.02 \psi\left(\theta_{r}\right)\right\}
$$

where

$$
x=\left[\begin{array}{c}
\theta_{l} \\
\omega_{l}
\end{array}\right], \quad A=\left[\begin{array}{cc}
0 & 1 \\
-2 & -0.24
\end{array}\right], \quad B=\left[\begin{array}{c}
0 \\
0.40
\end{array}\right] .
$$

Moreover, the normalized saturation function $\psi\left(\theta_{r}\right)$ is treated as a bounded unmeasurable disturbance by virtue of property 3 . It is worth recalling that, for our control design, only an upper bound on the deadzone spacing $\delta$ is required. The simulations were carried out with following robust control law:

$$
\begin{aligned}
\tau= & -\sigma_{2} \tilde{\omega}_{m}+\left(\theta_{m}-N_{0} \theta_{l}\right)-\rho_{m} \operatorname{Tanh}\left[(a+b t) \tilde{\omega}_{m}\right]+\chi_{2}\left(x, \tilde{\theta}_{m}, \hat{\beta}\right) \\
& +\rho_{l} \gamma_{2}\left(x, \tilde{\theta}_{m}, \hat{\beta}\right) \operatorname{Tanh}\left[(a+b t) \gamma_{2}\left(x, \tilde{\theta}_{m}, \hat{\beta}\right) \tilde{\omega}_{m}\right] .
\end{aligned}
$$

As a benchmark for comparison purposes, simulations were first carried out using the classical PD controller to evaluate the performance of the proposed robust adaptive compensators. The objective is a position maneuver of 1 radian (i.e. $\theta_{d}=1$ for a system with a sandwiched deadzone spacing of $\delta=0.02$ radians (close to 1.15 degrees). Figure 1 shows the performance of the two controllers for a step maneuver of $57.1^{\circ}$. It is evidently clear that the tracking performance of the adaptive controller outperforms the classical PD controller which is shown that in some cases leads to limit cycling. The load position tracking error defined as $\theta_{L}-\theta_{d}$ is shown in Figure 2 where error clearly approaches zero as the velocity $\omega_{l}$ approaches zero as predicted by the analysis. The control effort for a one radian trajectory is shown in Figure 3. In the analysis presented the deadzone nonlinearity itself was treated as a low level disturbance which allows the designer to increase gains as permitted by the experimental setup or application. In Figure 4(A) and Figure 4(B), the tracking performance and the tracking error of a desired trajectory $\theta_{d}=\sin (2 \pi t)$ are shown respectively. The motor angle $\theta_{m}$ and the motor velocity $\omega_{m}$ performances are demonstrated in Figure 5(A) and Figure 5(B) respectively. An abrupt vertical displacements in $\theta_{m}$ as well as a instantaneous increase in the angular velocity $\omega_{m}$ which acts to reduce the effect of the deadzone spacing on the load side angle $\theta_{l}$. The control effort for the sinusoidal reference trajectory is presented in Figure 6. The control effort moves aggressively in the beginning until the tracking error is reduced to a minimum values. Figure 7 demonstrate the evolution of the dynamic upper bounding adaptiation $\hat{\beta}$ which proofs its boundedness. 


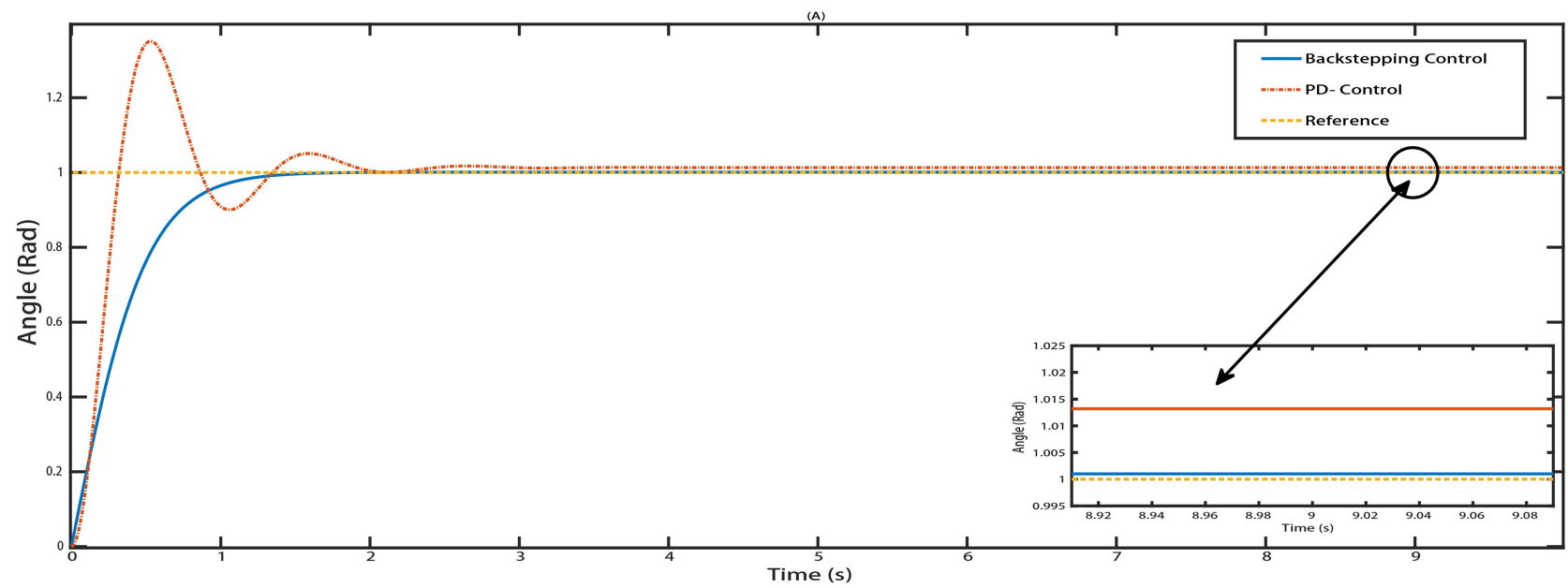

Figure 1. Load angle performance response for a 1.0 radian rotation maneuver under the robust adaptive control for system with sandwiched deadzone.

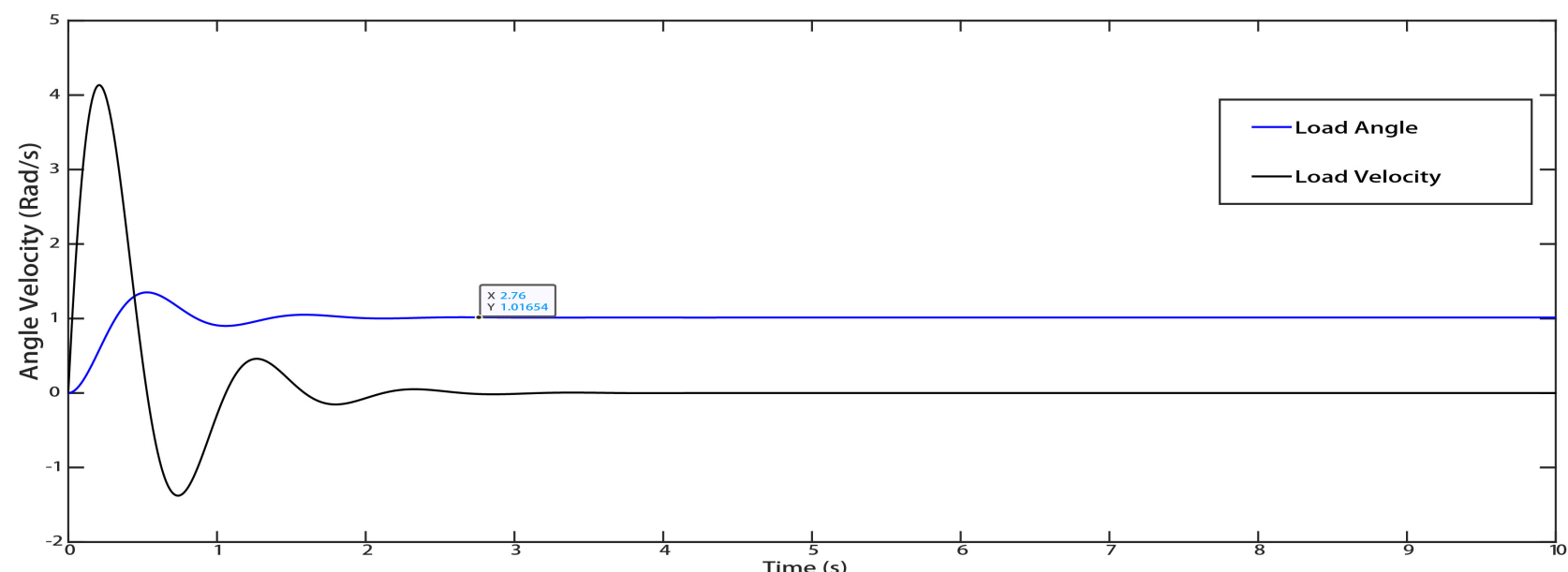

Figure 2. Load angle tracking performance response of the desired reference trajectory $\theta_{d}=\operatorname{Sin}(2 \pi t)$ under the robust adaptive control.

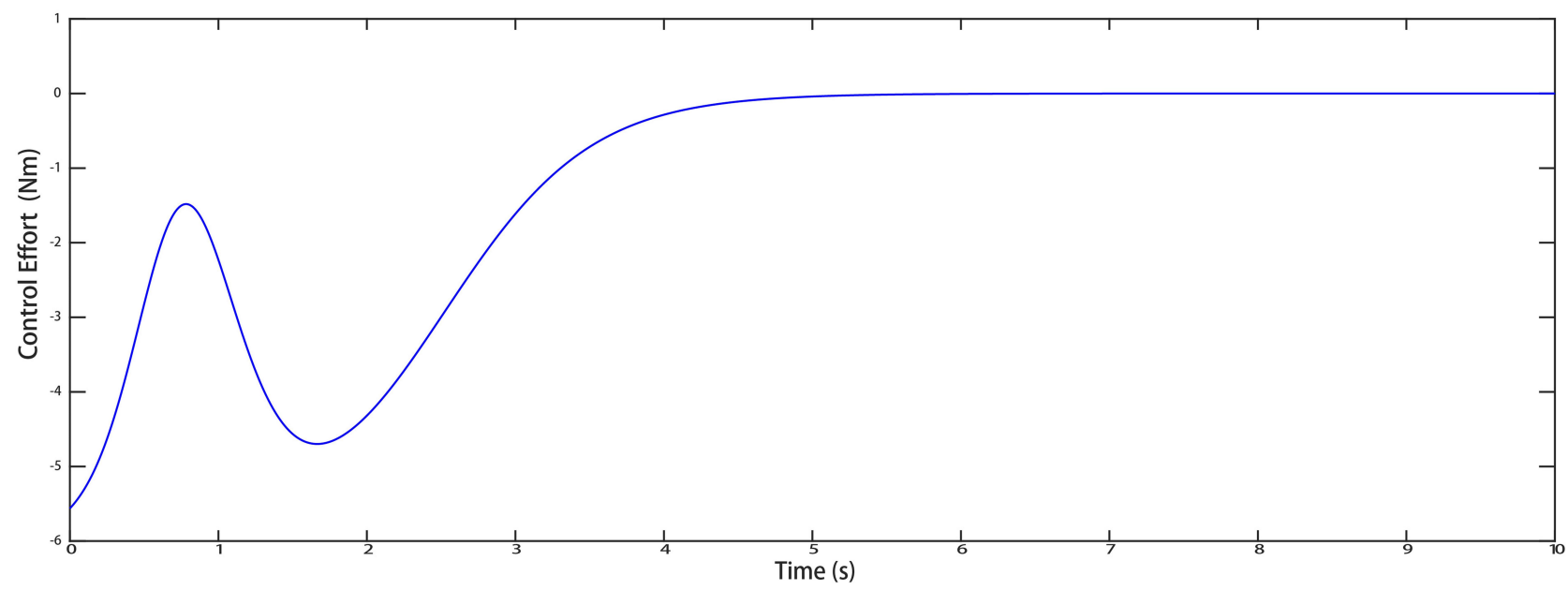

Figure 3. Control effort for a 1 radian maneuver by load angle $\theta_{L}$ performance. 

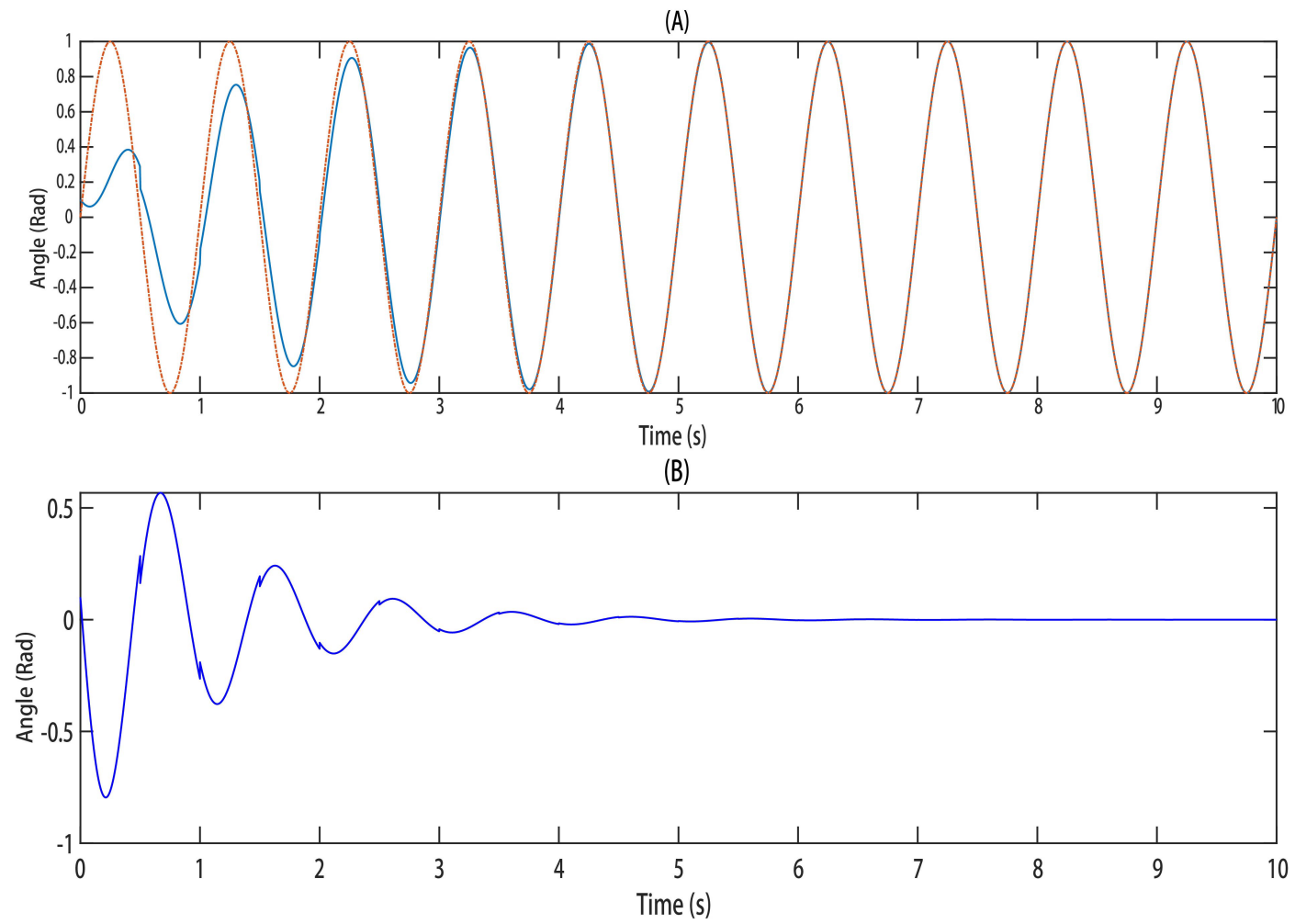

Figure 4. (A) Load angle tracking of a desired $\theta_{d}=\operatorname{Sin}(2 \pi t)$ under the robust adaptive control. (B) Load tracking error $\theta_{l}-\theta_{d}$.
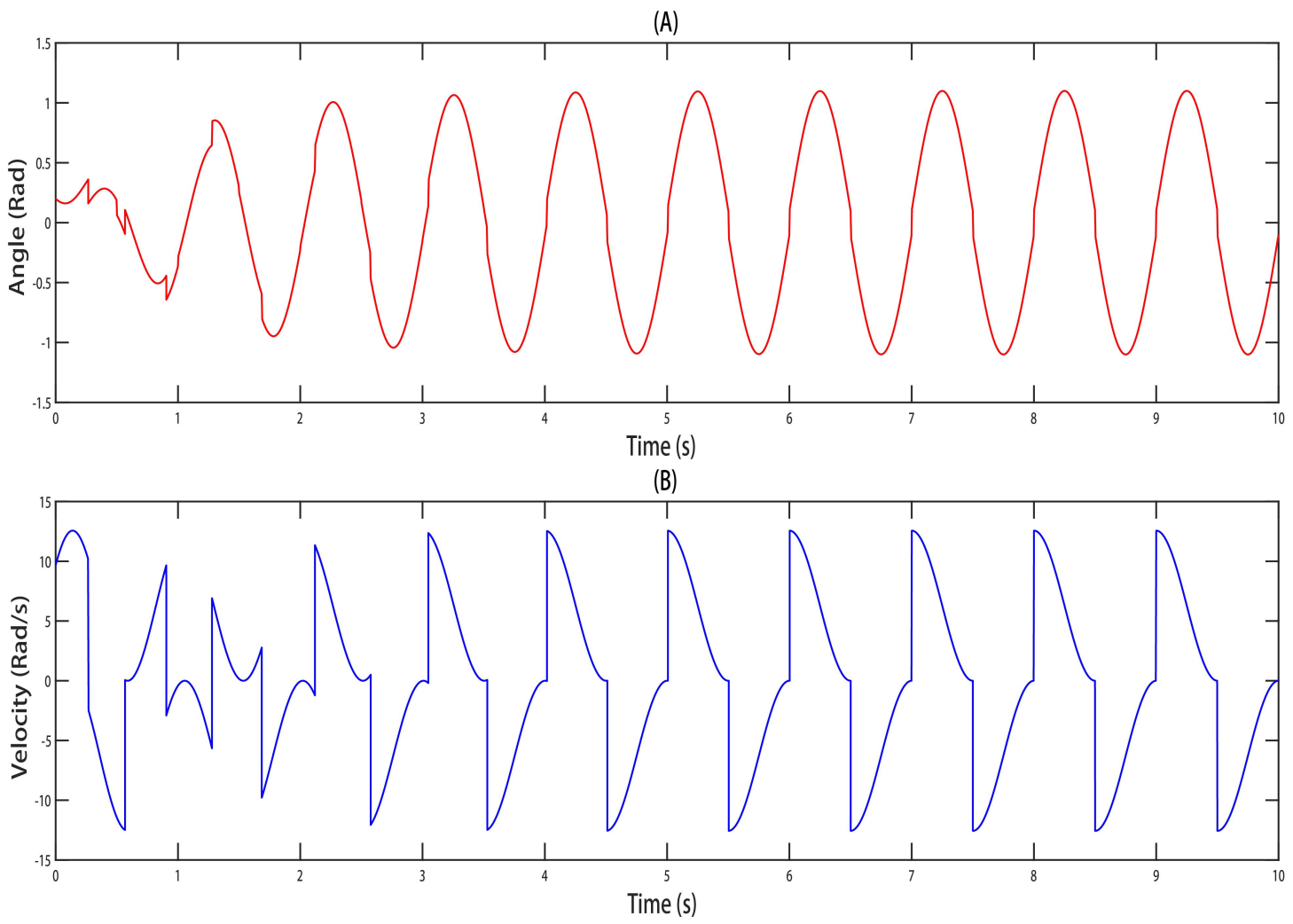

Figure 5. (A) Motor angle $\theta_{m}$ trajectory. (B) Motor angular velocity $\omega_{m}$. performance. 


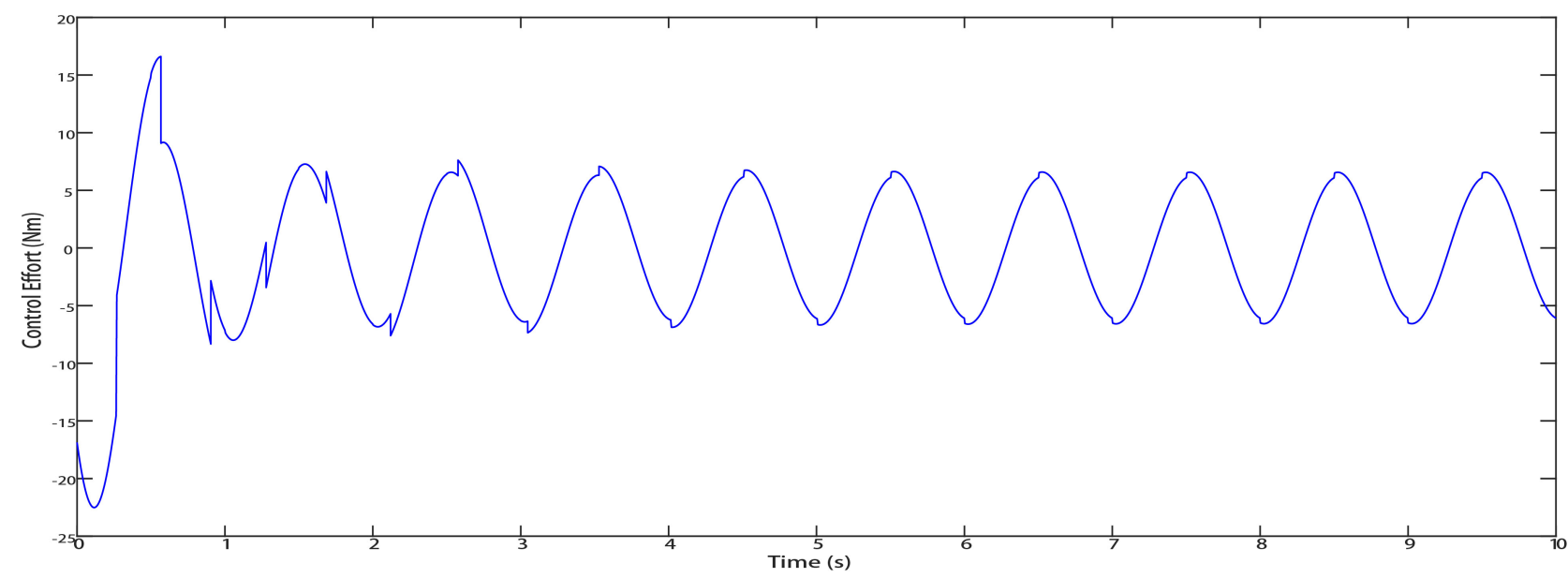

Figure 6. The robust adaptive control $\tau$.

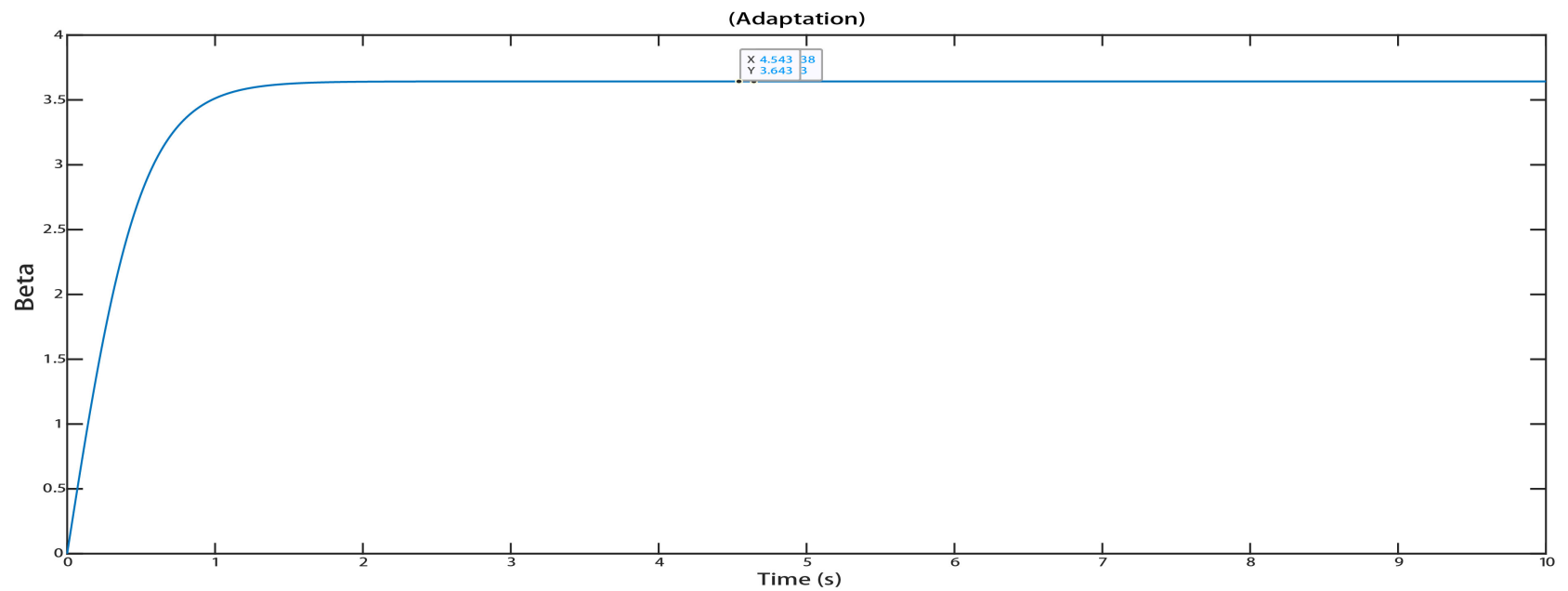

Figure 7. The evolution of the adaptation $\hat{\beta}$.

\section{Conclusion}

A robust adaptive backstepping compensator was employed for a dynamic system consisting of GTS drives which contain a compliant transmission with a deadzone nonlinearity. The new robust controller was designed to control the system with discontinuous deadzone in a sandwiched setting. The proposed controller did not require the deadzone nonlinearity's parameters. Only an upper estimate of the deadzone spacing is required. The advocated control design methodology has been proven to be robust to variations in the deadzone and inertia parameters and does not require exact knowledge of any of the deadzone parameters. Without the aforementioned model based cancellation, we are still achieving a good performance enhancement. Furthermore, the robustness of the controller to variations in the system parameters is being validated through extensive simulation and the results will be presented as they become finalized. For future work, extending the challenge to the case where the state dynamics are considered to be unmeasurable requiring a dynamic state observer to be de- 
signed.

\section{Acknowledgements}

The authors wish to acknowledge the support of the Public Authority for Applied Education and Training; (Project No. TS-16-009). Furthermore, the authors appreciate Dr. Lavanya Murugan's assistance in the simulations and analytical proofing of the theory development.

\section{Conflicts of Interest}

The authors declare no conflicts of interest regarding the publication of this paper.

\section{References}

[1] Shi, Z. and Zuo, Z. (2015) Backstepping Control for Gear Transmission Servo Systems with Backlash Nonlinearity. IEEE Transactions on Automation Science and Engineering, 12, 752-757. https://doi.org/10.1109/TASE.2014.2369430

[2] Ahmad, N., Alnaser, M., Sultan, E. and Alhendi, K. (2015) Robust Adaptive Control for a Class of Systems with Deadzone Nonlinearity. Intelligent Control and Automation, 6, 10-19. https://doi.org/10.4236/ica.2015.61002

[3] Recker, D., Kokotovic, P., Rhode, D. and Winkelman, J. (1991) Adaptive Nonlinear Control of Systems Containing a Deadzone. Proceedings of the 30 th IEEE Conference on Decision and Control, Brighton, 11-13 December 1991, 2111-2115. https://doi.org/10.1109/CDC.1991.261510

[4] Tao, G. and Kokotovic, P. (1993) Adaptive Control of Systems with Backlash. Automatica, 29, 323-335. https://doi.org/10.1016/B978-0-08-041717-2.50020-3

[5] Corradini, M.L., Orlando, G. and Parlangeli, G. (2004) A VSC Approach for the Robust Stabilization of Nonlinear Plants with Uncertain Nonsmooth Actuator Nonlinearities-A Unified Framework. IEEE Transactions on Automatic Control, 49, 807-813. https://doi.org/10.1109/TAC.2004.828324

[6] Shyu, K.-K., Hsu, K.-C. and Liu, W.-J. (2004) Variable Structure Control of Uncertain Nonlinear System with a Dead-Zone Input. IEEE International Conference on Networking, Sensing and Control, 2004, Taipei, 21-23 March 2004, 955-960. https://doi.org/10.1109/ICNSC.2004.1297076

[7] Corradini, M.L., Orlando, G. and Parlangeli, G. (2005) Robust Control of Nonlinear Uncertain Systems with Sandwiched Backlash. Proceedings of the 44th IEEE Conference on Decision and Control, Seville, 15-15 December 2005, 8112-8117. https://doi.org/10.1109/CDC.2005.1583475

[8] Stoorvogel, A.A., Wang, X., Saberi, A. and Sannuti, P. (2010) Stabilization of Sandwich Non-Linear Systems with Low-and-High Gain Feedback Design. Proceedings of the 2010 American Control Conference, Baltimore, 30 June-2 July 2010, 4217-4222. https://doi.org/10.1109/ACC.2010.5530848

[9] Saberi, A., Kokotovic, P. and Sussmann, H. (1990) Lobal Stabilization of Partially Linear Composite Systems. SIAM Journal on Control and Optimization, 28, 1491-1503. https://doi.org/10.1137/0328079

[10] Taware, A., Tao, G. and Teolis, C. (2002) Design and Analysis of a Hybrid Control Scheme for Sandwich Nonsmooth Nonlinear Systems. IEEE Transactions on Automatic Control, 47, 145-150. https://doi.org/10.1109/9.981735 
[11] Taware, A. and Tao, G. (2003) An Adaptive Dead-Zone Inverse Controller for Systems with Sandwiched Dead-Zones. International Journal of Control, 76, 755-769. https://doi.org/10.1080/0020717031000108776

[12] Taware, A., Tao, G. and Teolis, C. (2001) An Adaptive Dead-Zone Inverse Controller for Systems with Sandwiched Dead-Zones. Proceedings of the 2001 American Control Conference, Arlington, 25-27 June 2001, 2456-2461.

https://doi.org/10.1109/ACC.2001.946121

[13] Grip, H.F., Saberi, A., Stoorvogel, A.A., Wang, X. and Roy, S. (2010) Semiglobal Stabilization of Sandwich Systems by Dynamic Output Feedback. Proceedings of the 2010 American Control Conference, Baltimore, 30 June-2 July 2010, 4229-4234. https://doi.org/10.1109/ACC.2010.5530841

[14] Zhou, J., Wen, C. and Zhang, Y. (2004) Backstepping Control of a Class of Nonlinear Systems with Unknown Dead-Zone. IEEE Conference on Robotics, Automation and Mechatronics, Singapore, 1-3 December 2004, 513-518. https://doi.org/10.1109/RAMECH.2004.1438973

[15] Azhdari, M. and Binazadeh, T. (2021) Uniformly Ultimately Bounded Tracking Control of Sandwich Systems with Nonsymmetric Sandwiched Dead-Zone Nonlinearity and Input Saturation Constraint. Journal of Vibration and Control. https://doi.org/10.1177/1077546320987940

[16] Jain, S. and Khorrami, F. (1995) Robust Adaptive Control of a Class of Nonlinear Systems: State and Output Feedback. Proceedings of 1995 American Control Conference-ACC $95,3,1580-1584$. https://doi.org/10.1109/ACC.1995.529773

[17] Jain, S. and Khorrami, F. (1997) Decentralized Adaptive Control of a Class of Large Scale Interconnected Nonlinear Systems. IEEE Transactions on Automatic Control, 42, 136-154. https://doi.org/10.1109/9.554396 


\section{Appendix}

Step 0:

$$
\begin{gathered}
\theta_{m}^{*}=-\alpha B^{\mathrm{T}} P x-\hat{\beta} B^{\mathrm{T}} P x-\rho_{l} \operatorname{Tanh}\left[(a+b t) B^{\mathrm{T}} P x\right] \\
\dot{\tilde{\theta}}_{m}=\dot{\theta}_{m}-\dot{\theta}_{m}^{*}=\omega_{m}-\chi_{1}(x, \hat{\beta})-\gamma_{1} B \theta_{m}-\delta \psi\left(\theta_{r}\right) \\
\dot{\theta}_{m}^{*}=\frac{\partial \theta_{m}^{*}}{\partial x} \dot{x}+\frac{\partial \theta_{m}^{*}}{\partial \hat{\beta}} \dot{\hat{\beta}}+\frac{\partial \theta_{m}^{*}}{\partial t} \\
\frac{\partial \theta_{m}^{*}}{\partial x}=-\alpha B^{\mathrm{T}} P-\hat{\beta} B^{\mathrm{T}} P-\rho_{l} \operatorname{Sech}^{2}\left[(a+b t) B^{\mathrm{T}} P x\right] \cdot(a+b t) B^{\mathrm{T}} P \\
\frac{\partial \theta_{m}^{*}}{\partial \hat{\beta}}=-B^{\mathrm{T}} P x \\
\frac{\partial \theta_{m}^{*}}{\partial t}=-\rho_{l} \operatorname{Sech}^{2}\left[(a+b t) B^{\mathrm{T}} P x\right] \cdot b B^{\mathrm{T}} P x \\
\chi_{1}=\left\{-\alpha-\hat{\beta}-\rho(a+b t) \cdot \operatorname{Sech}^{2}\left[(a+b t) B^{\mathrm{T}} P x\right]\right\} B^{\mathrm{T}} P\left\{A x+B \theta_{m}\right\} \\
-\Gamma\left(B^{\mathrm{T}} P x\right)^{3}-\rho b B^{\mathrm{T}} P x \cdot \operatorname{Sech}^{2}\left[(a+b t) B^{\mathrm{T}} P x\right] \\
\gamma_{1}=\alpha B^{\mathrm{T}} P B+\hat{\beta} B^{\mathrm{T}} P B+\rho(a+b t) B^{\mathrm{T}} P B \cdot \operatorname{Sech}^{2}\left[(a+b t) B^{\mathrm{T}} P x\right]
\end{gathered}
$$

Step 1:

$$
\begin{aligned}
& \dot{\tilde{\omega}}_{m}=\dot{\omega}_{m}-\dot{\omega}_{m}^{*}=\dot{\omega}_{m}-\chi_{2}\left(x, \tilde{\theta}_{m}, \beta\right)-\gamma_{2}\left(x, \tilde{\theta}_{m}, \hat{\beta}\right) \operatorname{Sat}\left(\theta_{m}-N_{0} \theta_{l}\right) \\
& \omega_{m}^{*}=-\sigma_{0} \tilde{\theta}-2 B^{\mathrm{T}} P x-\chi_{1}(x, \hat{\beta})-\rho_{l} \gamma_{1}(x, \hat{\beta}) \operatorname{Tanh}\left[(a+b t) \gamma_{1} \tilde{\theta}\right] \\
& \dot{\omega}_{m}^{*}=\frac{\partial \omega_{m}^{*}}{\partial x} \dot{x}+\frac{\partial \omega_{m}^{*}}{\partial \hat{\beta}} \dot{\hat{\beta}}+\frac{\partial \omega_{m}^{*}}{\partial t} \\
& \dot{\tilde{\omega}}_{m}=\frac{1}{J_{m}}\left\{\tau-c_{m} \omega_{m}-\left(\theta_{m}-N_{0} \theta_{l}\right)+\operatorname{Sat}\left(\theta_{m}-N_{0} \theta_{l}\right)\right\}-\chi_{2}\left(x, \tilde{\theta}_{m}, \hat{\beta}\right) \\
& -\gamma_{2}\left(x, \tilde{\theta}_{m}, \hat{\beta}\right) \operatorname{Sat}\left(\theta_{m}-N_{0} \theta_{l}\right) \\
& \dot{\gamma}_{1}=\tau_{1}+\tau_{2} \cdot \operatorname{Sat}\left(\theta_{m}-N_{0} \theta_{l}\right)=\frac{\partial \gamma_{1}}{\partial x} \dot{x}+\frac{\partial \gamma_{1}}{\partial \hat{\beta}} \dot{\hat{\beta}}+\frac{\partial \gamma_{1}}{\partial t} \\
& \frac{\partial \gamma_{1}}{\partial x}=2 \rho(a+b t)^{2} B^{\mathrm{T}} P B \operatorname{Tanh}\left((a+b t) B^{\mathrm{T}} P x\right) \operatorname{Sech}^{2}\left[(a+b t) B^{\mathrm{T}} P x\right] B^{\mathrm{T}} P \\
& \frac{\partial \gamma_{1}}{\partial \hat{\beta}}=B^{\mathrm{T}} P B \dot{\hat{\beta}}=\Gamma B^{\mathrm{T}} P B\left\|B^{\mathrm{T}} P x\right\|^{2} \\
& \frac{\partial \gamma_{1}}{\partial t}=\rho b B^{\mathrm{T}} P B \cdot \operatorname{Sech}^{2}\left[(a+b t) B^{\mathrm{T}} P x\right] B+\rho(a+b t) B^{\mathrm{T}} P B \\
& \cdot \operatorname{Sech}^{2}\left[(a+b t) B^{\mathrm{T}} P x\right] B \operatorname{Tanh}\left[(a+b t) B^{\mathrm{T}} P x\right] \cdot b B^{\mathrm{T}} P x \\
& \tau_{1}=2 \rho(a+b t) B^{\mathrm{T}} P \operatorname{Tanh}\left((a+b t) B^{\mathrm{T}} P x\right) \operatorname{Sech}^{2}\left((a+b t) B^{\mathrm{T}} P x\right) \\
& \times(a+b t) B^{\mathrm{T}} P\left(A x+B \theta_{m}\right)+\frac{\partial \gamma_{1}}{\partial \hat{\beta}}+\frac{\partial \gamma_{1}}{\partial t}
\end{aligned}
$$




$$
\begin{aligned}
& \tau_{2}=2 \rho(a+b t) B^{\mathrm{T}} P \operatorname{Tanh}\left((a+b t) B^{\mathrm{T}} P x\right) \operatorname{Sech}\left((a+b t) B^{\mathrm{T}} P x\right)(a+b t) B^{\mathrm{T}} P B(\text { A.52 }) \\
& \dot{\chi}_{1}=\frac{\partial \chi_{1}}{\partial x} \dot{x}+\frac{\partial \chi_{1}}{\partial \hat{\beta}} \dot{\hat{\beta}}+\frac{\partial \chi_{1}}{\partial t}+\frac{\partial \chi_{1}}{\partial \theta_{m}} \dot{\theta}_{m} \\
& \frac{\partial \chi_{1}}{\partial x}=\left\{-2 \rho_{l}\left[(a+b t) B^{\mathrm{T}} P\right]^{2}\left\{A x+B \theta_{m}\right\}-2 \rho_{l} b(a+b t) B^{\mathrm{T}} P A-\rho_{l} b B^{\mathrm{T}} P\right\} \\
& \times \operatorname{Sech}^{2}\left((a+b t) B^{\mathrm{T}} P x\right) \operatorname{Tanh}\left[(a+b t) B^{\mathrm{T}} P x\right] \\
& -\left\{\rho_{l}(a+b t)\right\} B^{\mathrm{T}} P A \operatorname{Sech}^{2}\left((a+b t) B^{\mathrm{T}} P x\right) \\
& -\{\alpha+\hat{\beta}\} B^{\mathrm{T}} P A-3 \Gamma\left(B^{\mathrm{T}} P x\right)^{2} B^{\mathrm{T}} P \\
& \frac{\partial \chi_{1}}{\partial \hat{\beta}} \dot{\hat{\beta}}=-\Gamma B^{\mathrm{T}} P\left\{A x+B \theta_{m}\right\}\left\|B^{\mathrm{T}} P x\right\|^{2} \\
& \frac{\partial \chi_{1}}{\partial t}=-\rho_{l} b B^{\mathrm{T}} P\left\{A x+B \theta_{m}\right\} \operatorname{Sech}^{2}\left((a+b t) B^{\mathrm{T}} P x\right) \\
& -2 \rho_{l}\left\{(a+b t) B^{\mathrm{T}} P\left(A x+B \theta_{m}\right)+b^{2} B^{\mathrm{T}} P x\right\} \operatorname{Sech}^{2}\left((a+b t) B^{\mathrm{T}} P x\right) \\
& \times \operatorname{Tanh}\left[(a+b t) B^{\mathrm{T}} P x\right] \\
& \frac{\partial \chi_{1}}{\partial \theta_{m}} \dot{\theta}_{m}=B^{\mathrm{T}} P B \omega_{m}\left\{-\alpha-\hat{\beta}-\rho_{l}(a+b t) \operatorname{Sech}^{2}\left((a+b t) B^{\mathrm{T}} P x\right)\right\} \\
& \dot{\chi}_{1}=\tau_{3}+\tau_{4} \cdot \operatorname{Sat}\left(\theta_{m}-N_{0} \theta_{l}\right) \\
& \tau_{3}=\frac{\partial \chi_{1}}{\partial x}\left\{A x+B m\left(\theta_{m}-N_{0} \theta_{l}\right)\right\}+\frac{\partial \chi_{1}}{\partial \hat{\beta}}+\frac{\partial \chi_{1}}{\partial t}+\frac{\partial \chi_{1}}{\partial \theta_{m}} \dot{\theta}_{m} \\
& \tau_{4}=-\frac{\partial \chi_{1}}{\partial x} \cdot B \\
& \tau_{4}=-2 \rho_{l}(a+b t) B^{\mathrm{T}} P B\left\{(a+b t) B^{\mathrm{T}} P\left(A x+B \theta_{m}\right)+b B^{\mathrm{T}} P x\right\} \\
& +\rho_{l} B^{\mathrm{T}} P B\{(a+b t) A+b\} \operatorname{Sech}^{2}\left[(a+b t) B^{\mathrm{T}} P x\right] \\
& +(\alpha+\hat{\beta}) B^{\mathrm{T}} P A B+3 \Gamma\left(B^{\mathrm{T}} P x\right)^{2} B^{\mathrm{T}} P B
\end{aligned}
$$

Step 2:

$$
\begin{gathered}
\tau=-\tilde{\theta}_{m}-\sigma_{m} \tilde{\omega}_{m}-\frac{1}{J_{d}}\left(N_{0} \theta_{l}-\theta_{m}\right)-\chi_{2}+\rho_{m} \gamma_{2} \tanh \left[(a+b t) \gamma_{2} \tilde{\omega}_{m}\right] \\
-\rho_{l} \tanh \left[(a+b t) \tilde{\omega}_{m}\right] \\
\chi_{2}=-\sigma_{0} \omega_{m}+\sigma_{0} \chi_{1}-2 B^{\mathrm{T}} P\left\{A x+B m\left(\theta_{m}-N_{0} \theta_{l}\right)\right\} \\
-\rho_{m}+\tau_{3}-\rho_{l} \tau_{1} \operatorname{Tanh}\left[(a+b t) \gamma_{1} \tilde{\theta}_{m}\right] \\
-\rho_{l} \gamma_{l} \operatorname{Sech}^{2}\left[(a+b t) \gamma_{1} \tilde{\theta}_{m}\right]\left[b \gamma_{1} \tilde{\theta}_{m}+(a+b t) \tau_{1} \tilde{\theta}_{m}+(a+b t) \gamma_{1}\left(\omega-\chi_{1}\right)\right] \\
\gamma_{2}=\sigma \gamma_{1}+2 B^{\mathrm{T}} P B+\tau_{4}-\rho_{l} \tau_{2} \operatorname{Tanh}[(a+b t) \tilde{\theta}] \\
\quad-\rho_{l} \gamma_{1} \operatorname{Sech}^{2}\left[(a+b t) \gamma_{1} \theta_{m}\right]\left\{(a+b t) \tau_{2}-(a+b t) \gamma_{1}^{2}\right\}
\end{gathered}
$$

